\title{
Small Solar Wind Transients and Their Connection to the Large-Scale Coronal Structure
}

\author{
E.K.J. Kilpua $\cdot$ J.G. Luhmann · J. Gosling $\cdot$ Y. Li • \\ H. Elliott • C.T. Russell • L. Jian • A.B. Galvin • \\ D. Larson · P. Schroeder • K. Simunac · G. Petrie
}

Received: 5 December 2008 / Accepted: 1 April 2009 / Published online: 21 April 2009

(C) The Author(s) 2009. This article is published with open access at Springerlink.com

\begin{abstract}
It has been realized for some time that the slow solar wind with its embedded heliospheric current sheet often exhibits complex features suggesting at least partially transient origin. In this paper we investigate the structure of the slow solar wind using the observations by the Wind and STEREO spacecraft during two Carrington rotations (2054 and 2055). These occur at the time of minimum solar activity when the interplanetary medium is dominated by recurrent high-speed streams and large-scale interplanetary coronal mass ejections (ICMEs) are rare. However, the signatures of transients with small scale-sizes and/or low magnetic field strength (comparable with the typical solar wind value, $\sim 5 \mathrm{nT}$ ) are frequently found in the slow solar wind at these times. These events do not exhibit significant speed gradients across the structure, but instead appear to move with the surrounding flow.
\end{abstract}

STEREO Science Results at Solar Minimum

Guest Editors: Eric R. Christian, Michael L. Kaiser, Therese A. Kucera, O.C. St. Cyr.

E.K.J. Kilpua $(\varangle)$

Department of Physical Sciences, Theoretical Physics Division, University of Helsinki, Helsinki,

Finland

e-mail: emilia.kilpua@helsinki.fi

E.K.J. Kilpua · J.G. Luhmann · Y. Li · D. Larson · P. Schroeder

Space Sciences Laboratory, University of California, Berkeley, CA, USA

J. Gosling

Laboratory for Atmospheric and Space Physics, University of Colorado, Boulder, CO 80303, USA

H. Elliott

Southwest Research Institute, 6220 Culebra, San Antonio, TX 78238, USA

C.T. Russell · L. Jian

Institute of Geophysics and Planetary Physics, UCLA, Los Angeles, CA, USA

A.B. Galvin · K. Simunac

Institute for the Study of Earth, Oceans, and Space, University of New Hampshire, Durham, NH 03824, USA

G. Petrie

National Solar Observatory, 950 N Cherry Ave., Tucson, AZ 85719, USA 
Source mapping using models based on GONG magnetograms suggests that these transients come from the vicinity of coronal source surface sector boundaries. In situ they are correspondingly observed in the vicinity of high density structures where the dominant electron heat flux reverses its flow polarity. These weak transients might be indications of dynamical changes at the coronal hole boundaries or at the edges of the helmet streamer belt previously reported in coronagraph observations. Our analysis supports the idea that even at solar minimum, a considerable fraction of the slow solar wind is transient in nature.

\section{Introduction}

During the solar activity minimum the low-latitude heliosphere is characterized by recurrent high-speed solar wind streams that carry the heliospheric open field lines, often rooted in the low latitude extensions of the polar coronal holes or in large mid-to-low latitude holes. The origin of the slow $\left(<450 \mathrm{~km} \mathrm{~s}^{-1}\right)$ solar wind is, however, much less understood. The slow solar wind includes the features sometimes associated with the heliospheric plasma sheet (HPS), defined as a high density and high beta, relatively narrow $(\sim 0.01 \mathrm{AU}$ near the orbit at the Earth) region that contains the heliospheric current sheet (HCS). The HPS is thought to be at least in part connected with the closed field lines of the coronal streamer belt (Gosling et al., 1981). However, the slow solar wind spans a much larger region than suggested by the width of the coronal streamer belt, giving support to the idea that part of the slow solar wind originates from the coronal hole boundaries (e.g. Lionello et al., 2005). The detailed interpretation of the slow solar wind is also hampered by the frequent occurrence at $1 \mathrm{AU}$ of compressions between solar wind streams of high and low speed, where the medium may be altered from its original state.

Availability of high quality solar images over the last decade has reinforced the idea that coronal streamers are far from steady structures (Sheeley et al., 1997; Wang et al., 1998, 2000). Small blobs of enhanced density are observed to flow outward from the streamer cusps at the rate of about four blobs per day. In LASCO coronagraph images the blobs first become visible around 3-4 solar radii $\left(R_{\mathrm{S}}\right)$ with speeds ranging from 0 to $100 \mathrm{~km} \mathrm{~s}^{-1}$, and they accelerate to speeds of $250-400 \mathrm{~km} \mathrm{~s}^{-1}$ in the outer edge of the C3 field of view $\left(\sim 30 R_{\mathrm{S}}\right)$. The radial acceleration profile of the 'blobs' is consistent with a slow solar wind acceleration profile (Sheeley et al., 1997). The latest images from the STEREO Heliospheric Imagers (Sheeley et al., 2008a, 2008b; Rouillard et al., 2008, 2009) track these structures up to $1 \mathrm{AU}$ or more on occasion. However, it is not clear whether they are tracers of slow solar wind, or are simply the slow solar wind itself.

In interplanetary space the slow solar wind has a very variable structure, ranging from relatively smooth and featureless outflows to large-scale slow ICMEs (Interplanetary Coronal Mass Ejections). Crooker et al. (1996) and Kahler, Crooker, and Larson (2003) used observed magnetic fields and suprathermal electrons to argue that a complex layer of intertwined flux tubes was a good description of the HPS. Moldwin et al. (2000) presented evidence for small flux-rope-like features in the interplanetary field although they could not associate them with a particular solar wind type or solar origin.

Sheeley et al. (1997) had earlier suggested that the release of material by reconnection at coronal hole boundaries may be the mechanism that maintains their boundaries in the case of rigidly rotating holes. The most common type of reconnection, also found by Lionello et al. (2005) in a numerical simulation of evolving coronal hole boundaries, is the so-called interchange reconnection where an open field line reconnects with an adjacent closed field line. Other suggested reconfiguration mechanisms of coronal fields include closed loops, 
which expand into the solar wind. These loops can be regarded to become "open". Reconnection between the two oppositely oriented open field lines form an U-shaped magnetic structure, open to the outer heliosphere at both ends, which is quickly carried away in the solar wind, and loops that close down forming a streamer (Wang et al., 2000; Lionello et al., 2005). All of these processes are probably constantly occurring at the boundaries of the 'last' closed field lines of the coronal helmet streamer belt, as well as at the cusps of the helmet streamers. The question is whether the in situ observations of slow solar wind can be understood in these terms alone, or whether other slow solar wind sources such as rapidly diverging open fields (e.g. Wang and Sheeley, 1990) are major contributors.

For the present study, we surveyed the structure of the slow $\left(<450 \mathrm{~km} \mathrm{~s}^{-1}\right)$ solar wind observed during Carrington rotations 2054 and 2055 (March - April 2007) coinciding with the present solar activity minimum. These Carrington rotations exhibited exceptionally welldefined high speed streams separated by periods of $<450 \mathrm{~km} \mathrm{~s}^{-1}$ plasma flows with typical slow solar wind appearance.

The analysis makes use of the multipoint observations provided by Solar TErrestrial RElation Observatory (STEREO) (Kaiser et al., 2007). STEREO consists of two identical spacecraft, one that leads the Earth (STEREO A; STA) and one that lags the Earth (STEREO B; STB) on its orbit around the Sun. During periods of the investigation the angular separation of the STEREO spacecraft was between $1.3^{\circ}-5.6^{\circ}$, corresponding to a separation of $\sim 0.01-0.02 \mathrm{AU}$ in the east-west direction. This is significantly less than the typical dimensions of magnetic clouds at $1 \mathrm{AU}$ that have average radial diameters of $\sim 0.27 \mathrm{AU}$ at solar minimum (Jian, 2008) and similar magnetic field behavior has been reported for longitudinal separations of $\sim 10^{\circ}$ (Mulligan et al., 1999). The multi-view capability of the STEREO measurements was further enhanced by using the observations by the Wind and the Advanced Composition Explorer (ACE) spacecraft that at the time of the study were located at the Lagrangian point L1, approximately between the STEREO spacecraft. ACE and Wind thus measured very similar solar wind conditions and in this work we will use primarily Wind measurements to describe the conditions at L1. Our data sets come from the magnetometers, plasma analyzers (PLASTIC) and solar wind electron analyzers (SWEA) on STEREO described in Acuna et al. (2008), Galvin et al. (2008), and Sauvaud et al. (2008), respectively, the magnetometer and 3D plasma analyzer (3DP) on Wind (Lepping et al., 1995; Lin et al., 1995), and the plasma analyzer (SWEPAM) and plasma spectrometer (SWICS) on ACE.

Due to very low solar activity no large-scale, clear ICMEs were identified during this period, but we searched for intervals of any scale, lasting more than 1 hour, when classical signatures of ICMEs were present. The various magnetic field, solar wind plasma, plasma compositional and suprathermal particle signatures that distinguish ICMEs from the ambient solar wind are summarized and discussed e.g. by Gosling (1990), Neugebauer and Goldstein (1997), and Zurbuchen and Richardson (2006).

The ICME identification can be sometimes very ambiguous (Gosling, 1997) as there is no signature that would be present in all ICMEs and various signatures do not always occur simultaneously. We faced the same problem when identifying the small transients. We searched for the intervals with decreased magnetic field variability, smooth rotation of the magnetic field and clear decreases in the proton temperature $\left(T_{\mathrm{p}}\right)$ and proton beta. Solar wind temperature depressions are considered as one of the most reliably ICME identifiers (Gosling, Pizzo, and Bame, 1973; Richardson and Cane, 1995). Using the empirical correlation between solar wind speed and proton temperature (Lopez, 1987) to calculate the "expected proton temperature" $\left(T_{\mathrm{ex}}\right)$ Richardson and Cane (1995) found that ICMEs typically have $T_{\mathrm{p}} / T_{\mathrm{ex}}<0.5$. Their formula is calibrated for the Near-Earth Heliospheric data base (OMNI) and for the L1 measurements we calculated the $T_{\mathrm{p}} / T_{\mathrm{ex}}$ ratio using the OMNI 
data. For the STEREO spacecraft no such a formula has yet been determined and thus, for the STEREO data we searched for the intervals with clear depression of $T_{\mathrm{p}}$, requiring that the thermal speed is below $20 \mathrm{~km} \mathrm{~s}^{-1}$ (Russell and Shinde, 2003).

In addition, we examined suprathermal solar wind electrons with energies above $\sim 80 \mathrm{eV}$ to diagnose the magnetic connection to the Sun. We examine these data for signatures of counterstreaming electrons (indicating closed loops still attached to the Sun), heat-flux dropouts (indicating detached U field structure), unidirectional flow and false polarities (possibly indicating interchange reconnection).

ICMEs also exhibit various compositional anomalies including increased helium to proton ration $(\mathrm{He} / \mathrm{p}>0.08)$, enhanced $\mathrm{Fe}$ charge states and enhancement of $\mathrm{O}^{+7} / \mathrm{O}^{+6}$ (e.g. Richardson and Cane, 2004 and references therein). We investigated the above listed ratios and the Fe charge states for small transients identified at L1 using the ACE measurements and for the events observed at the location of the STEREO spacecraft the Fe charge states were studied.

\section{Observations}

Figure 1 shows the solar wind magnetic field and plasma measurements obtained during the Carrington rotations 2054 and 2055 from the 1-hour averaged OMNI data base. At the time of this study OMNI data was composed of the Wind and ACE spacecraft measurements, both located at L1. STEREO spacecraft were still close to the Sun - Earth line and the overall pattern of the solar wind parameters was very similar at the location of the STEREO spacecraft as at L1. The red-hatched regions in Figure 1 indicate the transients that were identified based on the smooth and organized magnetic field, clear drop in plasma beta and/or temperature as well as distinct suprathermal electron signatures. Tables $1-3$ summarize the key signatures of the transients at each spacecraft.

Table 1 (two last columns) also gives the estimated propagation speeds for each transient from Wind to the STA and STB (if observed). We have used the average solar wind speed during the transient, the time difference between the leading edge observations at different spacecraft and assumed the propagation along the GSE $X$-direction. For most of the events the estimated propagation speeds are in the range of the slow solar wind speed. Significant differences between the estimated propagation speeds and the transient's speeds (e.g. Event E5) arise presumably from the non-radial propagation as well as the uncertainties in determining the transient front boundaries. For one transient (Event E17) the leading edge was detected almost simultaneously at all spacecraft.

As typical for the solar minimum period Figure 1(b) shows the presence of recurring high speed solar wind structure in the near-Earth interplanetary medium during the investigated Carrington rotations. These streams emanated from two large equatorial coronal holes (Figure 2). It is seen from Figure 1 that transients constitute a significant, but not a dominant part of the slow solar wind during that period.

During two Carrington rotations we identified a total of 17 separate transient structures from the slow solar wind. Seven (E2, E4, E9, E10, E12, E14, E17) of these 17 events could be identified at all three locations and three events (E1, E3, E5) at two of the locations, i.e. they were detected by the Wind spacecraft and one of the STEREO spacecraft. The remaining eight events could be identified only at one of the locations. There were four events detected only by Wind (E7, E8, E11, E13), one event seen only by STA (E15) and two events detected only by STB (E6, E16).

Most of the identified transients exhibited several ICME signatures. An example of such an event is given in Figure 3 that displays the measurements during the transient on 


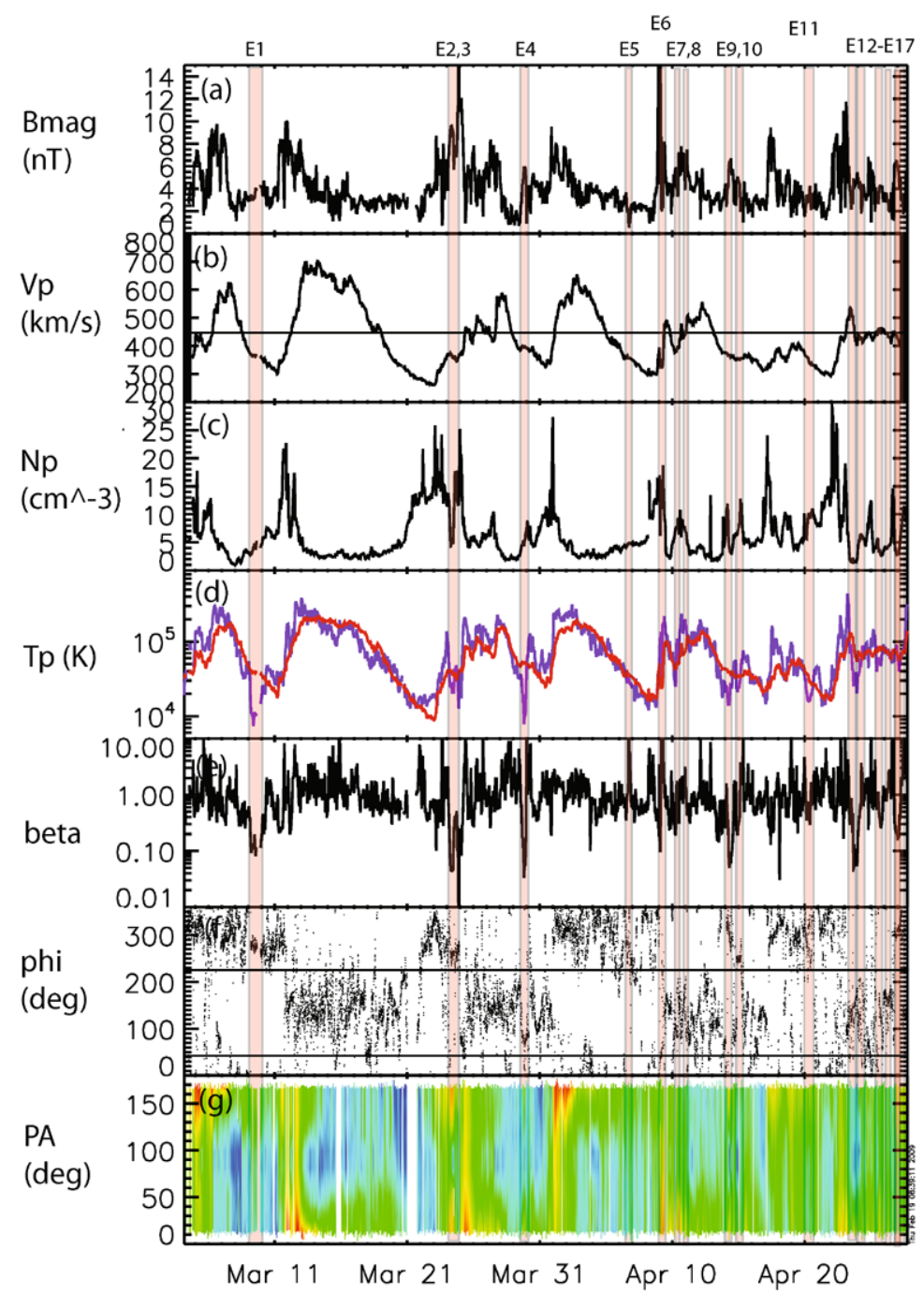

Figure 1 Measurements from the OMNI data base during the Carrington rotations 2054 and 2055. Panels show: (a) magnetic field magnitude, (b) solar wind speed (horizontal line indicates the speed $450 \mathrm{~km} \mathrm{~s}^{-1}$ ), (c) proton density, (d) proton temperature (purple line) and the temperature expected for the observed solar wind speed (red line; Richardson and Cane, 1995), (e) proton beta, (f) magnetic field GSE azimuth angle $(\phi)$ and $(\mathrm{g})$ the pitch angle spectrogram of suprathermal $260 \mathrm{eV}$ electrons from the Wind spacecraft (data shifted to the bow shock using the time shift from the OMNI data base). Two horizontal lines $\left(\phi=45^{\circ}\right.$ and $\left.\phi=225^{\circ}\right)$ in panel (f) indicate the division to away $\left(\phi\right.$ within $\left.45^{\circ}-225^{\circ}\right)$ and toward $\left(\phi=315^{\circ}\right.$ on average) magnetic sectors. The colored regions show the identified transients.

29 March 2007 (Event E4) that was seen by all observing spacecraft. At this time STEREO spacecraft were separated by only 2.8 degrees. This structure had a diameter in the radial direction less than $0.1 \mathrm{AU}\left(\sim 2350 R_{E}\right)$ and low magnetic field magnitude $(<6 \mathrm{nT})$, but as seen from Figure 3, several clear ICME signatures were present: smooth magnetic field profile, organized magnetic field behavior, depressed proton beta and temperature as well as 


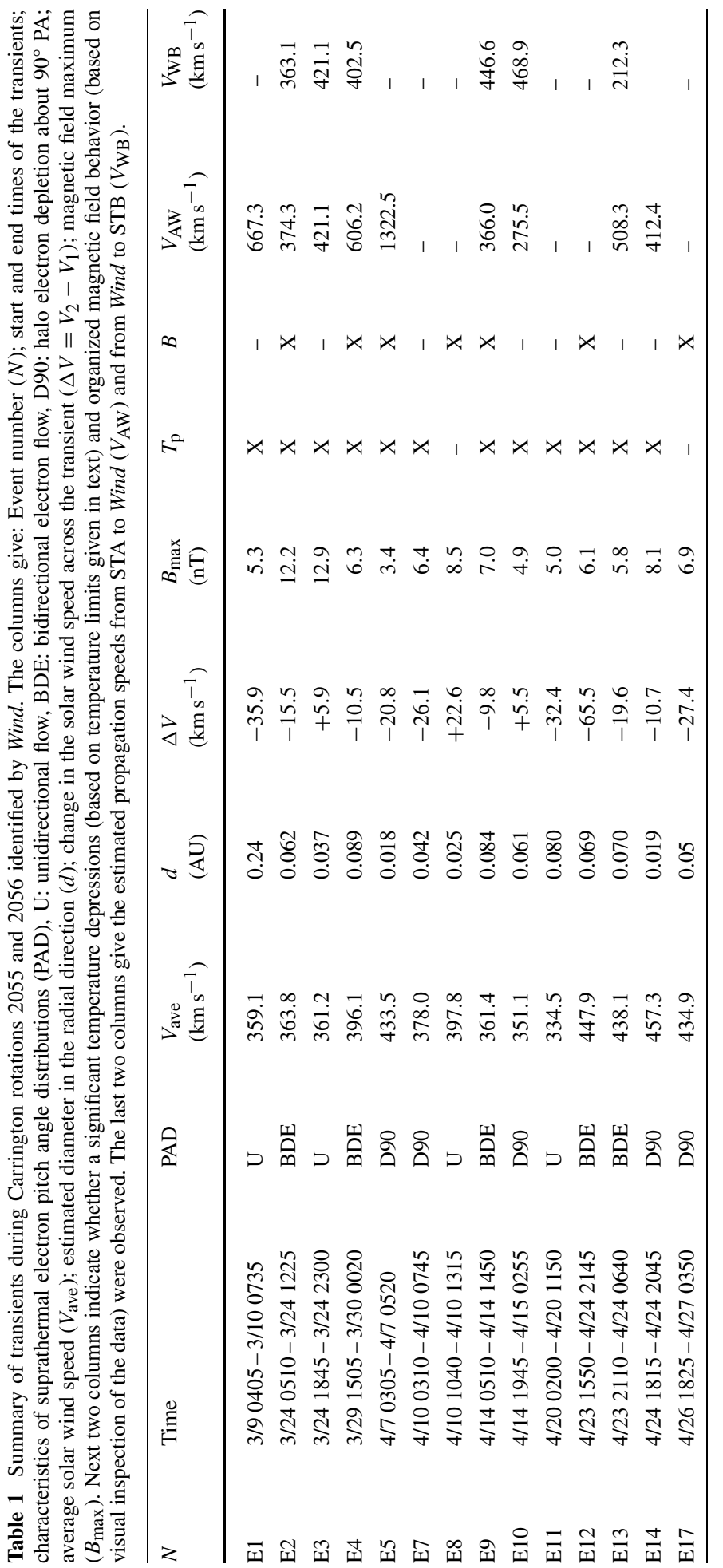


Table 2 Summary of transients during Carrington rotations 2055 and 2056 identified by the STA spacecraft. The panels are same as in Table 1.

\begin{tabular}{lllllllll}
\hline$N$ & Time & PAD & $\begin{array}{l}V_{\text {ave }} \\
\left(\mathrm{km} \mathrm{s}^{-1}\right)\end{array}$ & $\begin{array}{l}d \\
(\mathrm{AU})\end{array}$ & $\begin{array}{l}\Delta V \\
\left(\mathrm{~km} \mathrm{~s}^{-1}\right)\end{array}$ & $\begin{array}{l}B_{\max } \\
(\mathrm{nT})\end{array}$ & $T_{\mathrm{p}}$ & $\mathrm{B}$ \\
\hline E1 & $3 / 90300-3 / 100515$ & $\mathrm{U}$ & 366.5 & 0.23 & -28.5 & 5.6 & $\mathrm{X}$ & - \\
E2 & $3 / 240235-3 / 240933$ & BDE & 368.7 & 0.062 & -16.0 & 12.1 & $\mathrm{X}$ & $\mathrm{X}$ \\
E4 & $3 / 291320-3 / 292050$ & BDE & 386.9 & 0.086 & -6.0 & 5.7 & $\mathrm{X}$ & $\mathrm{X}$ \\
E5 & $4 / 70212-4 / 70814$ & $?$ & 346.6 & 0.05 & +13.3 & 3.4 & $\mathrm{X}$ & - \\
E9 & $4 / 140130-4 / 141220$ & BDE & 376.3 & 0.05 & +10.8 & 6.3 & - & $\mathrm{X}$ \\
E10 & $4 / 141450-4 / 142150$ & D90 & 361.2 & 0.061 & +5.0 & 6.9 & - & - \\
E12 & $4 / 231827-4 / 232150$ & BDE & 498.5 & 0.041 & -13.2 & 4.8 & - & $\mathrm{X}$ \\
E14 & $4 / 241605-4 / 241820$ & $\mathrm{U}$ & 450.2 & 0.026 & +1.4 & 7.5 & - & $\mathrm{X}$ \\
E15 & $4 / 250930-4 / 251455$ & BDE & 475.8 & 0.062 & -21.2 & 4.1 & - & $\mathrm{X}$ \\
E17 & $4 / 261950-4 / 271030$ & BDE & 418.9 & 0.15 & -8.6 & 5.9 & - & $\mathrm{X}$ \\
\hline
\end{tabular}

Table 3 Summary of transients during Carrington rotations 2055 and 2056 identified by the STB spacecraft.

\begin{tabular}{llllllrll}
\hline$N$ & Time & PAD & $\begin{array}{l}V_{\text {ave }} \\
\left(\mathrm{km} \mathrm{s}^{-1}\right)\end{array}$ & $\begin{array}{l}d \\
(\mathrm{AU})\end{array}$ & $\begin{array}{l}\Delta V \\
\left(\mathrm{~km} \mathrm{~s}^{-1}\right)\end{array}$ & $\begin{array}{l}B_{\max } \\
(\mathrm{nT})\end{array}$ & $T_{\mathrm{p}}$ & $\mathrm{B}$ \\
\hline E2 & $3 / 240835 ?-3 / 241700$ & BDE & 361.0 & 0.073 & -8.2 & 14.2 & $\mathrm{X}$ & $\mathrm{X}$ \\
E3 & $3 / 242145-3 / 250405$ & D90 & 368.0 & 0.056 & +23.8 & 14.6 & - & $\mathrm{X}$ \\
E4 & $3 / 291820-3 / 300412$ & BDE & 386.7 & 0.091 & -2.8 & 5.9 & $\mathrm{X}$ & $\mathrm{X}$ \\
E6 & $4 / 90610-4 / 90840$ & $\mathrm{U}$ & 300.3 & 0.018 & -50.0 & 19.8 & $?$ & $\mathrm{X}$ \\
E9 & $4 / 140840-4 / 141725$ & BDE & 360.8 & 0.076 & -5.3 & 5.9 & $\mathrm{X}$ & $\mathrm{X}$ \\
E10 & $4 / 142305-4 / 140925$ & D90 & 354.2 & 0.088 & -7.2 & 5.7 & - & - \\
E14 & $4 / 240455-4 / 240935$ & BDE & 409.3 & 0.040 & -26.1 & 4.5 & - & - \\
E16 & $4 / 260750-4 / 261545$ & D90 & 393.4 & 0.075 & -2.6 & 5.8 & - & $\mathrm{X}$ \\
E17 & $4 / 262012-4 / 270820$ & D90 & 427.1 & 0.12 & -14.1 & 6.2 & - & $\mathrm{X}$ \\
\hline
\end{tabular}

clear counterstreaming of heat flux electrons. All spacecraft recorded very similar features and it is clear that they encountered the same transient.

Figure 4 shows an example of the transient on 14 April 2007 (Event E9) that was encountered by all spacecraft, but when clear differences were visible at different locations. The separation between the STEREO spacecraft was 4.1 degrees. Wind and STB that were only 1.2 degrees from each other recorded very similar magnetic field and plasma characteristics while STA measurements show distinct differences in the magnetic field profile. Pitch angle (PA) spectrograms show the strahl centered on PA $180^{\circ}$ before the transient. During the transient all spacecraft detected counterstreaming electrons. The transient E10 (also visible in Figure 4) that occurred close to the Event E9 is distinguished by depressed proton temperature and beta. This latter transient was associated with a clear depletion in the halo electron distribution functions centered on $90^{\circ}$ PA (e.g. Gosling, Skoug, and Feldman, 2001).

The transient shown in Figure 5 exhibit magnetic cloud-like behavior at STA (left panel): During this transient the magnetic field profile was smooth and symmetric, peaking approximately in the middle of the transient and the magnetic field direction rotated in an organized way. Proton beta and temperature were slightly depressed. The separation between the STA and STB was 5.3 degrees and no signatures of the transient could be identified at STB. At 
Figure 2 EUVI image at $195 \AA$ by STA/SECCHI/EUVI on 25 March 2007 at 03:01 UT. The image features two large equatorial coronal holes.

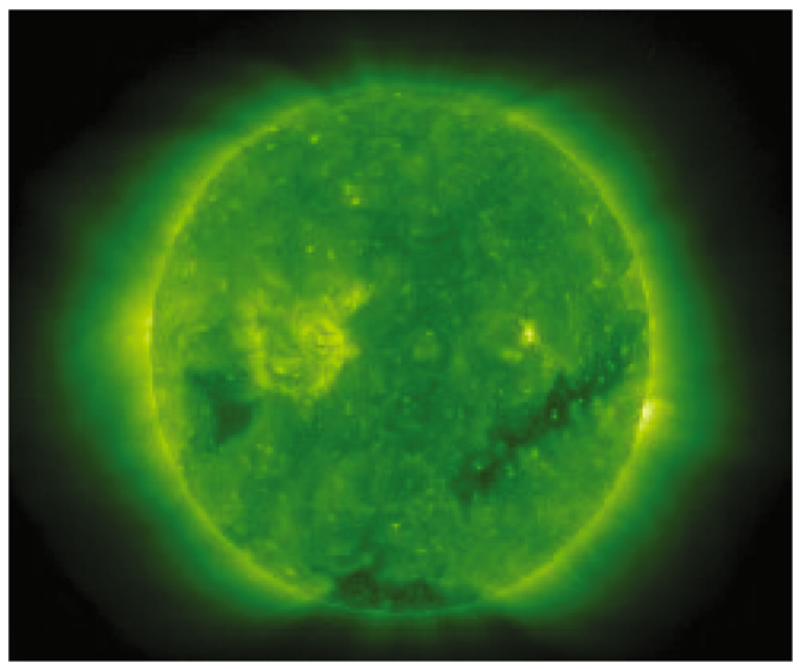

Wind, located 3.7 degrees away from STA, there was a short period of depressed proton beta and temperature. PA spectrograms at STA show strahl appearing at PA $180^{\circ}$ throughout the transient while at Wind a halo depletion around $90^{\circ}$ PA can be distinguished. In addition the field angles were clearly different. Although characteristics were very different between Wind and STA the timing considerations (Table 1) suggest that the spacecraft could have indeed encountered the same transient. This event was also one of the smallest in our data set, having a radial diameter of only $0.019 \mathrm{AU}$ at STA.

Three events (E1, E7 and E11) were associated with only a clear temperature drop. However, temperature depressions are considered one of the best indicators of the CME related material in the solar wind (e.g. Gosling, Pizzo, and Bame, 1973; Richardson and Cane, 1995). An example of a temperature depression event observed by Wind is given in Figure 6 (Event E11). STA and STB located 3.4 and 1.4 degrees away, respectively at this time, did not observe the associated temperature depression. Figure 6 shows that at the time of the event the magnetic field strength was low $(<5 \mathrm{nT})$ and the magnetic field directional changes appear very disorganized. The PA spectrogram indicates that the suprathermal electron flux was unidirectional throughout this transient, making it unlikely that it was a closed magnetic structure.

Table 4 lists the average values of the magnetic field maximum $\left(B_{\max }\right)$, the speed change across the transient $(|\Delta V|)$ and the radial diameter of all identified transients. We have also included in Table 4 the corresponding values for the 36 ICMEs identified during the solar minimum years 1995 - 1997 and for the 272 ICMEs identified over solar cycle 23 (19952006) by Jian et al. (2006), Jian (2008). On average the magnetic field magnitudes during the intervals in Tables $1-3$ were close to the nominal solar wind value, $\sim 5 \mathrm{nT}$. All except two transients had $B_{\max }<10 \mathrm{nT}$ and the average $B_{\max }$ for all identified transients was $7.3 \mathrm{nT}$. The $B_{\max }$ reported for the solar minimum ICMEs was almost twice as large, $13.9 \mathrm{nT}$.

The third panel of Table 4 indicates that in general the solar wind speed did not change significantly during the passages of the identified transients; they appear to be carried along with the surrounding slow solar wind. As seen from Table 4 solar wind speed variations across the ICME were generally lower at solar minimum than when averaged over all activity phases. However, the observed $|\Delta V|$ for small transients, $20 \mathrm{~km} \mathrm{~s}^{-1}$, is still significantly smaller than the average for solar minimum ICMEs, $80 \mathrm{~km} \mathrm{~s}^{-1}$. In addition, none 


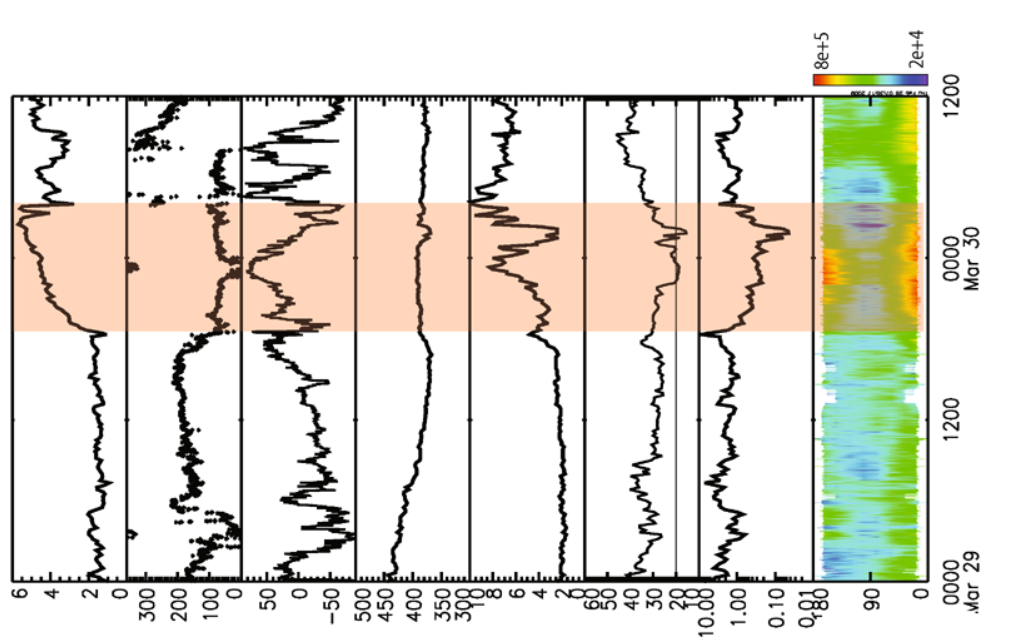

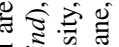

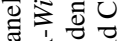

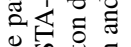

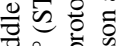

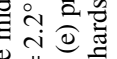

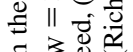

$\Xi$ 政

$\stackrel{0}{0} \sum_{0}^{\circ}$

泮高

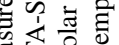

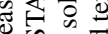

의 $\overline{0}$

$\forall \infty$ is

䨌 II

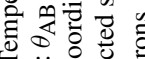

- बं ठ ठ

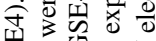

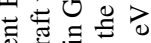

\%

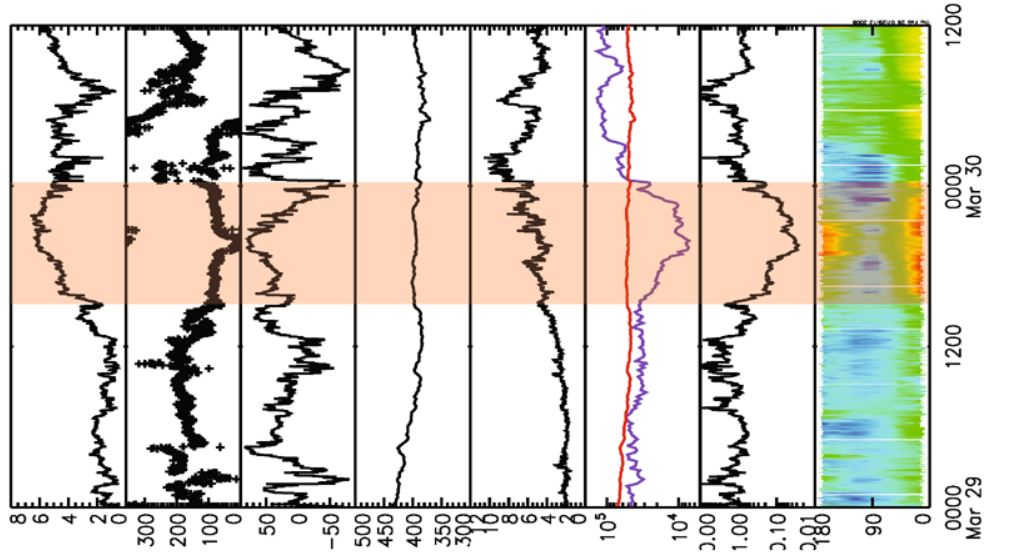

记

웜의

등

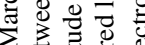

施

ते

함ㅎㅁ

एक

可

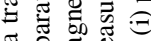

o 屯্

氙包可

0 过造

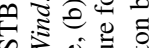
녕

흐을

ठี

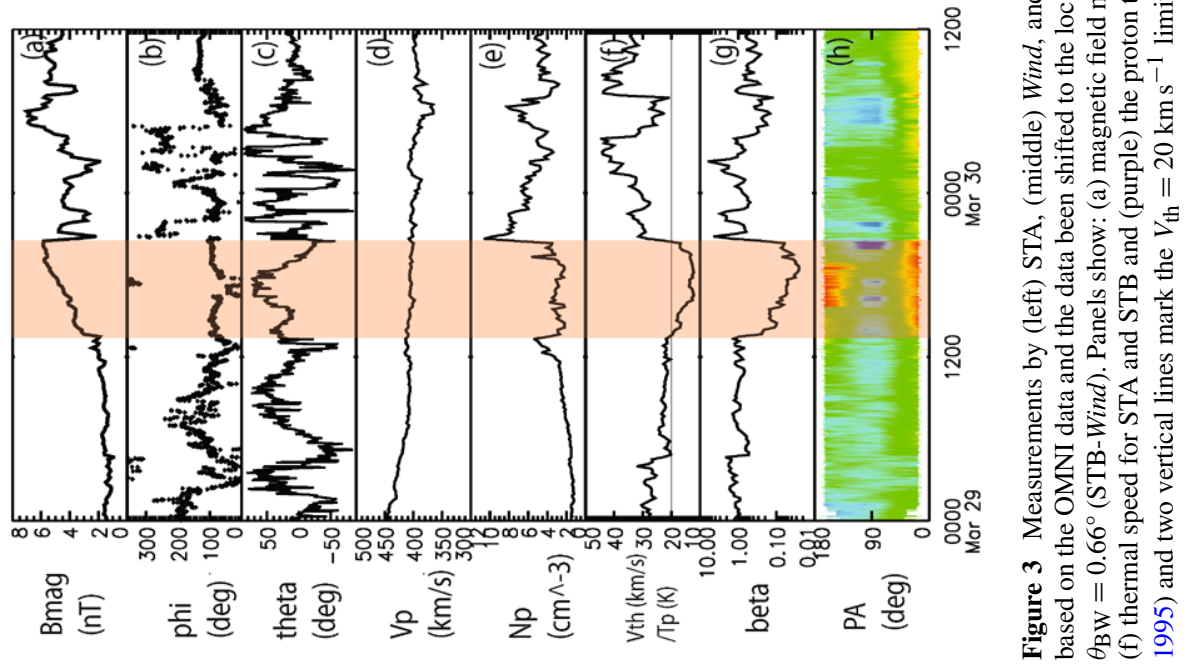




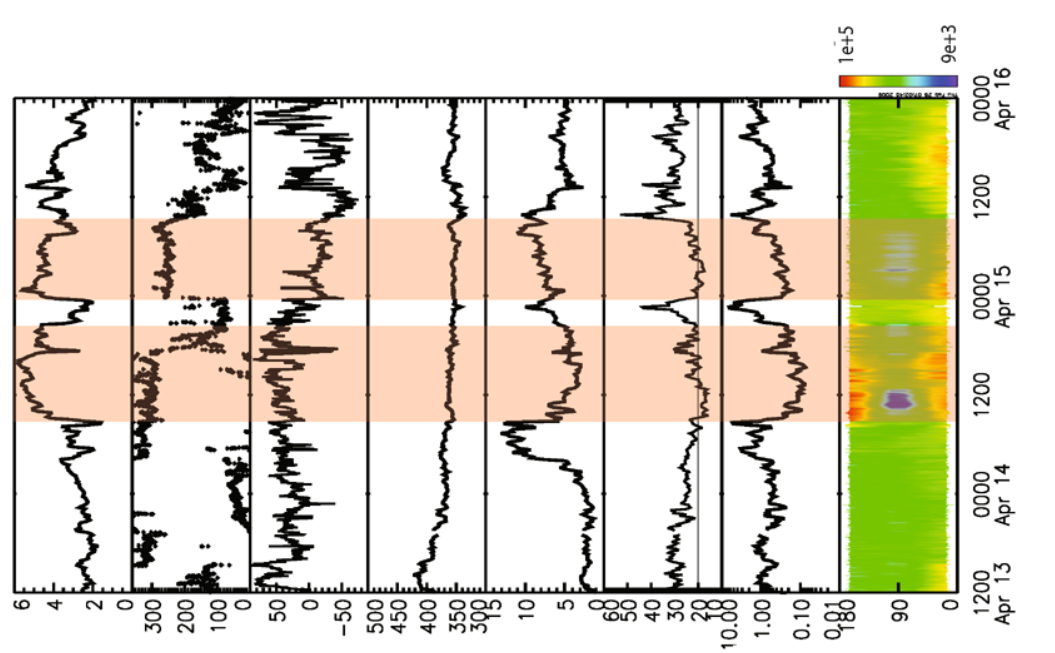

$\cong$ $\sum_{0}^{0}$
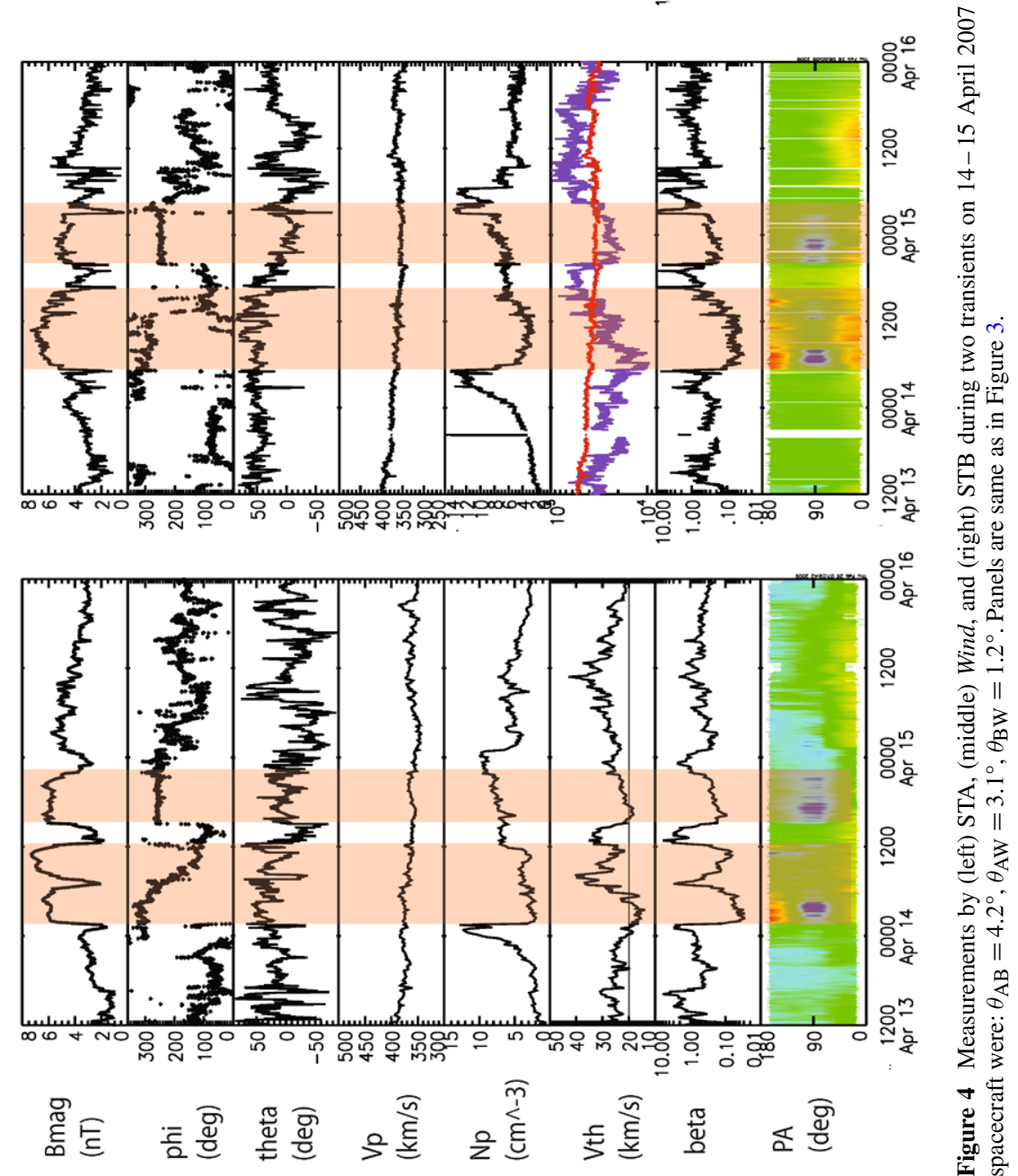

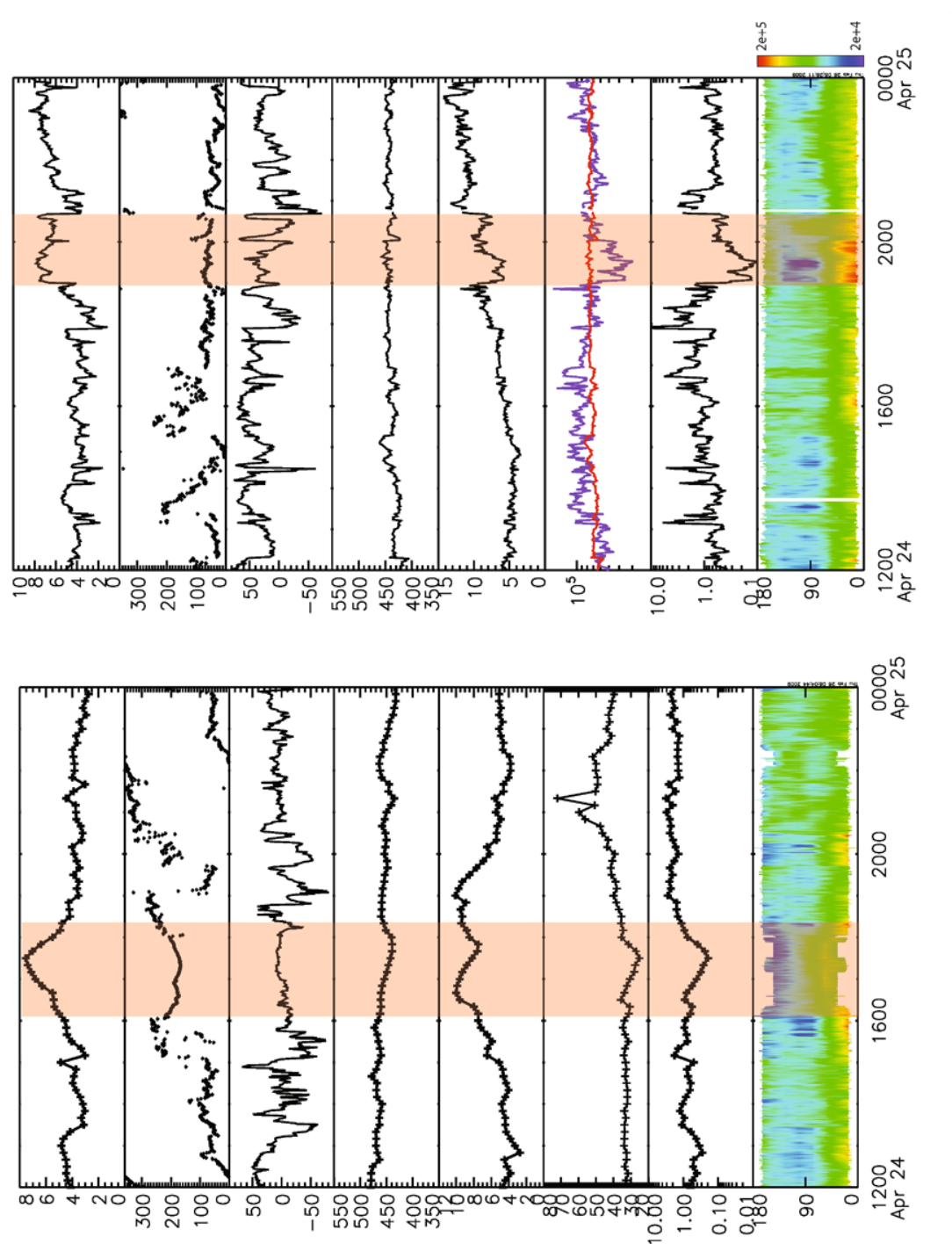

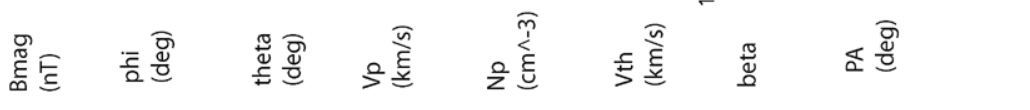

in

in

$\frac{\infty}{0^{2}}$

$\ddot{0}$

言

$\stackrel{2}{ \pm}$

อี

$\sum_{0}^{\infty}$

8

을 
Figure 6 Measurements by

Wind during a transient on 8 April 2007 (Events E11). The separation angles between the spacecraft were: $\theta_{\mathrm{AB}}=4.8^{\circ}$, $\theta_{\mathrm{AW}}=3.4^{\circ}, \theta_{\mathrm{BW}}=1.4^{\circ}$. Panels are same as in Figure 3.

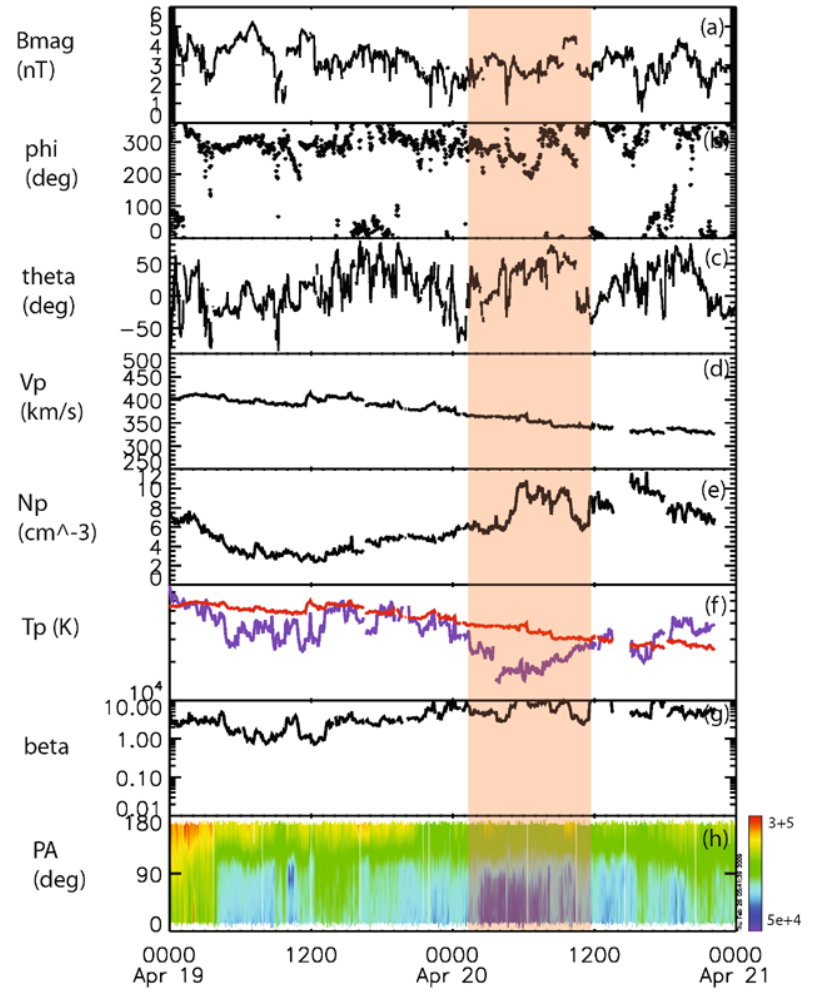

Table 4 Average values of magnetic field maximum, speed change and the radial diameter (a) for all 17 transients in our study; (b) for large-scale ICMEs identified near 1 AU by Jian et al. (2006) and Jian (2008) at solar minimum (years 1995-1997); (c) for large-scale ICMEs identified near 1 AU during 1995-2006 by Jian et al. (2006) and Jian (2008). The numbers in parenthesis give the associated probable errors of the mean.

\begin{tabular}{llccl}
\hline & Period & $B_{\max }(\mathrm{nT})$ & $|\Delta V|\left(\mathrm{km} \mathrm{s}^{-1}\right)$ & $d(\mathrm{AU})$ \\
\hline (a) & our study & $7.3( \pm 1.0)$ & $20( \pm 4)$ & $0.072( \pm 0.012)$ \\
(b) & Jian et al./min & $13.9( \pm 1.5)$ & $80( \pm 12)$ & $0.27( \pm 0.03)$ \\
(c) & Jian et al./all & $19.1( \pm 0.70)$ & $154( \pm 9)$ & $0.40( \pm 0.01)$ \\
\hline
\end{tabular}

of the small transients drove an interplanetary shock ahead of them. The fraction of shockassociated ICMEs was also found to be low near solar minimum and in 1996 none of the five observed ICMEs drove a shock (Jian et al., 2006).

Radial diameters of the identified transients, which we expect are produced by minor to modest coronal changes, ranged from $0.018\left(\sim 420 R_{E}\right)$, to 0.23 AU with average of 0.072 AU. All except two events (E1, E17) had radial diameters less than $0.1 \mathrm{AU}$. As a comparison solar minimum ICMEs had considerably larger radial diameters, about $0.27 \mathrm{AU}$ on average (Jian, 2008).

Seven of the 17 intervals listed in Table 1 were associated with clear counterstreaming suprathermal electrons and for five events strong electron halo depletions centered at $90^{\circ}$ PA were observed. The remaining events exhibited predominantly unidirectional heat flux 
flow. We also searched for the periods of false polarities (e.g. Kahler, Crooker, and Gosling, 1996) in which the magnetic field direction imply polarities opposite to those determined from the heat-flux directions and the periods exhibiting nearly isotropic dropouts of electron heat flux (McComas et al., 1989). However, we did not find indications of such intervals.

We also investigated solar wind compositional characteristics during the transients. Solar wind helium to proton ratio was investigated using the ACE-/SWEPAM data, the $\mathrm{O}^{+7} / \mathrm{O}^{+6}$ ratio using the ACE/SWICS data and finally the average iron charge states were studied from ACE/SWICS and STEREO/PLASTIC measurements. The $\mathrm{He} / \mathrm{p}$ and $\mathrm{O}^{+7} / \mathrm{O}^{+6}$ ratios did not show any significant increases during the transients. The average Fe charge states were also about the nominal solar wind values, except during the events E2 and E3 slightly enhanced charge states were observed at all spacecraft.

During Carrington rotations 2054 and 2055 the solar activity was minimal. Before 24 April 2007, covering the period that could affect the solar wind near 1 AU during the investigated period NOAA activity reports listed only four B class flares. The LASCO catalogue (Yashiro et al., 2004) listed 253 CMEs but the majority of these were reported as very poor events and/or events that were very narrow (few tens of degrees) and slow. No CMEs spanning over $100^{\circ}$, or halo CMEs, were observed. At the same time, the SECCHI Heliospheric Imagers observed the continuous outflow of apparently inhomogeneous material from the vicinity of the streamer belt (e.g. Sheeley et al., 2008a).

We examined both SOHO and STEREO SECCHI images to determine if there were any obvious changes at the mapped locations several days before the event times in Tables $1-3$, without success. However, since the investigated events were all very small/weak, specific related CMEs may have been too faint or narrow to be detected by the coronagraphs, or were not identifiable in the generally present complex outflows. We also examined the SOHO EIT and STEREO EUVI movies for coronal dimmings and post-flare loops that are regarded as good indicators of CMEs. The only clear dimming we were able to identify was on $25 \mathrm{March}$ thus being a good candidate to cause Event E3 (Figure 3). A very small coronal dimming in a suitable time window to produce another observed in situ disturbance on 14-15 April occurred 10 April.

Since the proposed transients seem to be embedded in the slow solar wind without associated speed enhancements, one should obtain a relatively reliable estimation of their solar launch time by using the average solar wind speed to track them from the location of the observing spacecraft back to the Sun. Figure 7(a) shows the Global Oscillation Network Group (GONG) magnetogram-based coronal field source surface model map with dashed vertical lines indicating the estimated "solar times" of the transients listed in Tables $1-3$. These models allow the approximate tracing of near-ecliptic solar wind back to its coronal source regions, as indicated by the coronal field lines connecting positive (green) and negative (red) polarity open fields to the ecliptic. It appears that most of the transients trace back to the vicinity of the model sector boundaries, which occur where the polarity of the open field lines mapped to the equator (approximately the ecliptic) change sign (see the Figure 7(a) caption). In Figure 7(b) we show the footpoints of coronal field lines that, according to the model, undergo a net opening or closing between these Carrington rotations and the prior rotations. The latter may provide some indication of where transients are likely to have arisen in the process of coronal hole boundary evolution. These regions, which trace the boundaries of the coronal holes or open fields, may represent the rough locations where the observed transients are born, if they result from reconnections near the helmet streamer base as it adjusts to the changing photospheric field. The mapping of these regions to the source surface of the model in Figure 7(c) suggests where they would begin their propagation into the solar wind. This belt of inferred transient fields is approximately the width of the slow 


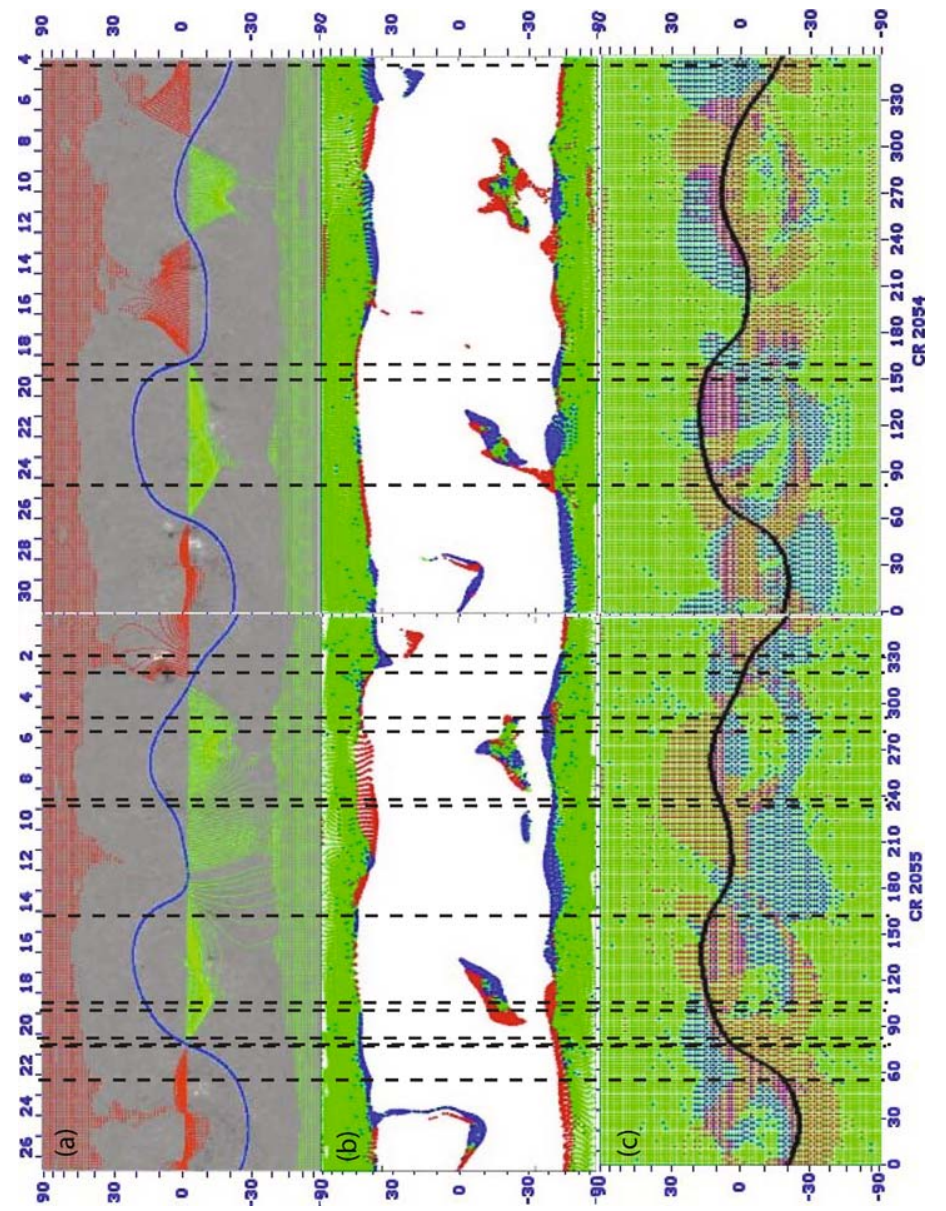

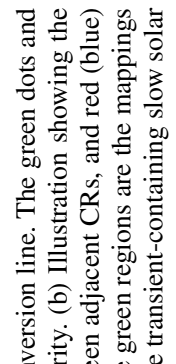

蛋造导 중하웡

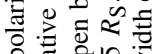
. 巳 $\cong$ 光 䒕纯

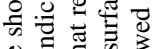
๑. $\Xi$ क क त

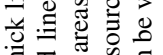

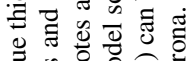

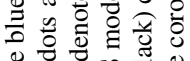

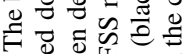

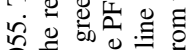

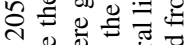

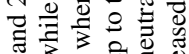

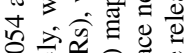

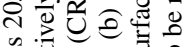

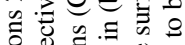

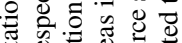
흔

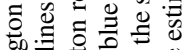

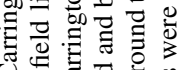
Uึ

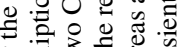
후월 드월

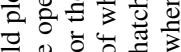

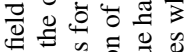

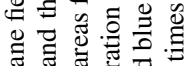

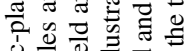
象导总

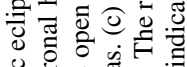

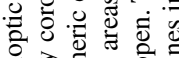

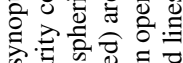
क

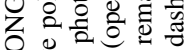
记

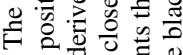

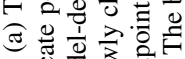

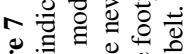

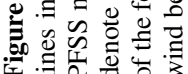


solar wind belt derived from in situ studies (e.g. Zhao and Hundhausen, 1983) (although the PFSS model with radial field at the source surface will tend to overestimate its latitudinal width).

\section{Discussion}

We have surveyed the structure of the slow solar wind during two Carrington rotations (2054 and 2055) coinciding with the solar activity minimum. As expected, due to very low solar activity no large-scale ICMEs were identified. However, the observations described here show that small and weak transients were frequently (17 in total) embedded in the slow solar wind.

It is documented that during solar minimum the large-scale ICMEs tend to be smaller and weaker than the ICMEs during solar maximum (e.g. Jian et al., 2006, 2008). However, the events investigated in this study were in general even smaller and weaker than solar minimum ICMEs; the identified transients had smaller scale sizes, smaller speed changes and lower magnetic field magnitudes than the average solar minimum ICME at $1 \mathrm{AU}$ (Table 4).

The investigated transients seemed to simply float with the slow solar wind: None of the events drove an interplanetary shock and most of the events did not exhibit significantly declining speed, which would have been an indication of an expanding structure. ICMEs near solar minimum do not generally drive shocks either and they are associated with smaller speed variations than ICMEs near solar maximum (Jian et al., 2006, 2008). The lack of declining speed profiles implies that the identified temperature depressions are rather inherent than a consequence of the expansion of the transient. We also failed to identify any ICME associated solar wind composition anomalies, during the small transients. However, the lack of composition anomalies is not surprising since the anomalies are most commonly found in fast ICMEs and ICMEs that exhibit flux rope structure (Richardson and Cane, 2004).

We observed a rather continuous range of scale sizes from very small transients, only a few hundreds of $R_{E}$ (comparable with the scale size of the "mini flux ropes" discussed by Moldwin et al. (2000) to the typical scale sizes of magnetic clouds at 1 AU (fraction of AU). As also discussed by Moldwin et al. (2000) this raises the question of whether these transients share a common origin, or are partly related to reconnection in the corona while others result from more distributed reconnection in the heliospheric current sheet. Similar to large-scale ICMEs the identified small transients exhibited very variable structures and characteristics.

The speeds and scale sizes of the studied events are consistent with what could be expected for the in situ counterparts of the coronal "blobs" reported first by Sheeley et al. (1997) that accelerated to the speed of the slow solar wind in the outer part of the LASCO C3 field of view. The SECCHI HI images (Sheeley et al., 2008a, 2008b; Rouillard et al., 2008) show that these blobs continue into the heliospheric space that maps back to the vicinity of the coronal streamers. Rouillard et al. (2009) [this issue] studied the evolution of the plasma compressed in-side the corotating interaction region (CIR) by combining the SECCHI HI images by STB to the in situ observations by STA. They reported a small-scale magnetic cloud with a radial extent of $0.08 \mathrm{AU}$, i.e. very similar to the average radial extent of transients studied in this work, merged with a CIR.

Over a decade ago, Crooker et al. (1993) proposed that the features associated with the so-called heliospheric plasma sheet were in fact interpretable as small-scale intertwined flux ropes compressed and distended along the heliospheric current sheet surface. Some of our 
events occurred just at the sector boundary, while others could only be inferred to be close to a sector boundary (see Figure 7). If Crooker's region of intertwined flux tubes, perhaps supporting local reconnection in the current sheets between flux tubes, is as wide as the slow wind belt, one would expect to see some transients with direct HCS associations, but more would be found distributed throughout this mixed-polarity boundary layer between solar wind sources with opposing magnetic fields.

Potential field source surface models suggest that the majority of the investigated events can be traced to the vicinity of coronal sector boundaries (Figure 7(a)). This raises a question whether these transients could be in situ counterparts of the material released by reconnection at the streamer cusps and near coronal hole boundaries. This picture is also inferred in Figures $7(\mathrm{~b}, \mathrm{c})$ from the potential field model, from coronagraph images by Sheeley et al. (1997), and from numerical simulations by Lionello et al. (2005) of the coronal response to differential rotation-related photospheric field evolution.

The suprathermal electron observations indicated that 7 out of 17 events were associated with counterstreaming electrons suggesting a closed magnetic field structure still connected to the Sun at both ends while the rest of the events exhibited unidirectional flow or strong depletion halo electron distributions concentrated about PA $90^{\circ}$. Unidirectional heat flow can be an indicator of interchange reconnection, while $90^{\circ}$ depletions are commonly observed on closed field lines of interplanetary coronal mass ejections and can arise from double connection to the Sun (Gosling et al., 2002). We did not find either heat flux drop outs that might imply fully detached plasmoids, or the false polarities which might be expected in interchange reconnection debris. This is consistent with a general rarity of such signatures (e.g. McComas et al., 1989).

The in situ observations were obtained from three spacecraft separated by only a few degrees during the investigation period. However, they did not always encounter the same transient, and when they did, if the spacecraft separation was more than few degrees the characteristics could be quite different at each location, suggesting that the longitudinal scale sizes of these transients are significantly smaller than the typical scale-sizes of CMErelated magnetic clouds near $1 \mathrm{AU}$ during which similar field behavior can be identified about at least 10 degrees of spacecraft separation (Mulligan et al., 1999).

The slow solar wind varies considerably with angular separation, consistent with the idea that it is a highly structured and complex entity. It is an interesting question what is the connection between the observed and the coronal "blobs" reported first by Sheeley et al. (1997) that accelerated to the speed of the slow solar wind in the outer part of the LASCO C3 field of view. The SECCHI HI images (Sheeley et al., 2008a, 2008b; Rouillard et al., 2008, 2009) show that these blobs continue into the heliospheric space that maps back to the vicinity of the coronal streamers. The later phase of the STEREO mission with larger angular separation offers intriguing new possibilities to study the connection between coronal dynamics and in situ transients. With one STEREO spacecraft one can image specific coronal outflows at the limb and try to connect them with the in situ observations at the other spacecraft. We intend to pursue this investigation as the mission progresses.

Acknowledgements Support for the STEREO mission solar and in situ data processing and analysis was provided through NASA contracts to the IMPACT, PLASTIC and SECCHI teams (the IMPACT contract to University of California Berkeley NAS5-03131, the PLASTIC contract to University of New Hampshire NAS5-00132, and the SECCHI contract to Naval Research Laboratory DPRS-13631-Y). Work on STEREO PLASTIC was funded under NASA contract NAS5-00132. This work was supported in part by the National Aeronautics and Space Administration under grant NAS5-00133. We thank R. Lepping for the WIND Magnetic Field Investigation data, R.P. Lin for the WIND 3D-plasma data and K.W. Ogilvie for the WIND SWE data. This work utilizes data obtained by the Global Oscillation Network Group (GONG) Program, managed by the National Solar Observatory, which is operated by AURA, Inc. under a cooperative agreement with 
the National Science Foundation. The data were acquired by instruments operated by the Big Bear Solar Observatory, High Altitude Observatory, Learmonth Solar Observatory, Udaipur Solar Observatory, Instituto de Astrofísica de Canarias, and Cerro Tololo Interamerican Observatory. The OMNI data were obtained through the NSSDC CDAWEB online facility. The SOHO LASCO CME catalog is generated and maintained at the CDAW Data Center by NASA and The Catholic University of America in cooperation with the Naval Research Laboratory. SOHO is a project of international cooperation between ESA and NASA.

Open Access This article is distributed under the terms of the Creative Commons Attribution Noncommercial License which permits any noncommercial use, distribution, and reproduction in any medium, provided the original author(s) and source are credited.

\section{References}

Acuna, M.H., Curtis, D., Scheifele, J.L., Russell, C.T., Schroeder, P., Szabó, A., Luhmann, J.G.: 2008, The STEREO/IMPACT magnetic field experiment. Space Sci. Rev. 136, $203-226$.

Crooker, N.U., Siscoe, G.L., Shodhan, S., Webb, D.F., Gosling, J.T., Smith, E.J.: 1993, Multiple heliospheric current sheets and coronal streamer belt dynamics. J. Geophys. Res. 101, 2467-2474.

Crooker, N.U., Burton, M.E., Phillips, J.L., Smith, E.J., Balogh, A.: 1996, Heliospheric plasma sheets as small-scale transients. J. Geophys. Res. 101, 2467-2474.

Galvin, A.B., Kistler, L.M., Popecki, M.A., et al.: 2008, The Plasma and Suprathermal Ion Composition (PLASTIC) investigation on the STEREO observatories. Space Sci. Rev. 136, 437-486.

Gosling, J.T.: 1990, Coronal mass ejections and magnetic flux ropes in interplanetary space. In: Priest, E.R., Lee, L.C., Russell, C.T. (eds.) Physics of Magnetic Flux Ropes, Geophys. Monogr. 58, AGU, Washington, $343-364$.

Gosling, J.T.: 1997, Coronal mass ejection: an overview. In: Crooker, N., Joselyn, J.A., Feynman, J. (eds.) Coronal Mass Ejections, Geophys. Monogr. 99, AGU, Washington, 245.

Gosling, J.T., Pizzo, V., Bame, S.J.: 1973, Anomalously low proton temperatures in the solar wind following interplanetary shock waves - evidence for magnetic bottles? J. Geophys. Res. 78, 2001 - 2009.

Gosling, J.T., Skoug, R.M., Feldman, W.C.: 2001, Solar wind electron halo depletions at $90^{\circ}$ pitch angle. Geophys. Res. Lett. 28, $4155-4158$.

Gosling, J.T., Borrini, G., Asbridge, J.R., Bame, S.J., Feldman, W.C., Hansen, R.T.: 1981, Coronal streamers in the solar wind at 1 AU. J. Geophys. Res. 86, 5438-5448.

Gosling, J.T., Skoug, R.M., Feldman, W.C., McComas, D.J.: 2002, Symmetric suprathermal electron depletions on closed field lines in the solar wind. Geophys. Res. Lett. 29, 14-1. CiteID 1573.

Jian, L.: 2008, Radial evolution of large-scale solar wind structures, Ph.D. Thesis, Univ. California, Los Angeles.

Jian, L., Russell, C.T., Luhmann, J.G., Skoug, R.M.: 2006, Properties of interplanetary coronal mass ejections at one AU during 1995-2004. Solar Phys. 239, 393-436.

Kahler, S.W., Crooker, N.U., Gosling, J.T.: 1996, The topology of intrasector reversals of the interplanetary magnetic field. J. Geophys. Res. 101, 24373-24382.

Kahler, S.W., Crooker, N.U., Larson, D.E.: 2003, Probing the magnetic polarity structure of the heliospheric current sheet. J. Geophys. Res. 108. CiteID 1316.

Kaiser, M., Kucera, T.A., Davila, J.M., St. Cyr, O.C., Guhathakurta, M., Christian, E.: 2007, The STEREO mission: an introduction. Space Sci. Rev. 136, 5 - 16. doi:10.1007/s11214-007-9277-0.

Lepping, R.P., Acuna, M.H., Burlaga, L.F., Farrell, W.M., Slavin, J.A., Schatten, K.H., Mariani, F., Ness, N.F., Neubauer, F.M., Whang, Y.C., Byrnes, J.B., Kennon, R.S., Panetta, P.V., Scheifele, J., Worley, E.M.: 1995, The Wind magnetic field investigation. Space Sci. Rev. 71, $207-229$.

Lin, R.P., Anderson, K.A., Ashford, S., Carlson, C., Curtis, D., Ergun, R., Larson, D., McFadden, J., McCarthy, M., Parks, G.K., Reme, H., Bosqued, J.M., Coutelier, J., Cotin, F., D’Uston, C., Wenzel, K.-P., Sanderson, T.R., Henrion, J., Ronnet, J.C., Paschmann, G.: 1995, A three-dimensional plasma and energetic particle investigation for the Wind spacecraft. Space Sci. Rev. 71, 125-153.

Lionello, R., Riley, P., Linker, J.A., Mikic, Z.: 2005, The effects of differential rotation on the magnetic structure of the solar corona: magnetohydrodynamic simulations. Astrophys. J. 625, 463-473.

Lopez, R.E.: 1987, Solar cycle invariance in solar wind proton temperature relationships. J. Geophys. Res. 92, $11189-11194$.

McComas, D.J., Gosling, J.T., Phillips, J.L., Bame, S.J., Luhmann, J.G., Smith, E.J.: 1989, Electron heat flux dropouts in the solar wind - evidence for interplanetary magnetic field reconnection? Solar Phys. 94, 6907-6916. 
Moldwin, M.B., Ford, S., Lepping, R., Slavin, J., Szabo, A.: 2000, Small-scale magnetic flux ropes in the solar wind. Geophys. Res. Lett. 27, 57-60.

Mulligan, T., Russell, C.T., Anderson, B.J., Lohr, D.A., Rust, D., Toth, B.A., Zanetti, L.J., Acuna, M.H., Lepping, R.P., Golsing, J.T.: 1999, Intercomparison of NEAR and Wind interplanetary coronal mass ejection observations. J. Geophys. Res. 104, 28217. doi:10.1029/1999JA900215.

Neugebauer, M., Goldstein, R.: 1997, Particle and field signatures of coronal mass ejections in the solar wind. In: Crooker, N., Joselyn, J.A., Feynman, J. (eds.) Coronal Mass Ejections, Geophys. Monogr. 99, AGU, Washington, 245.

Richardson, I.G., Cane, H.V.: 1995, Regions of abnormally low proton temperature in the solar wind (19651991) and their association with ejecta. J. Geophys. Res. 100, 23397.

Richardson, I.G., Cane, H.V.: 2004, Identification of interplanetary coronal mass ejections at 1 AU using multiple solar wind plasma composition anomalies. J. Geophys. Res. 109, A09104. doi:10.1029/ 2004JA010598.

Rouillard, A.P., Davies, J.A., Forsyth, R.J., Rees, A., Davis, C.J., Harrison, R.A., Lockwood, M., Bewsher, D., Crothers, S.R., Eyles, C.J., Hapgood, M., Perry, C.H.: 2008, First imaging of corotating interaction regions using the STEREO spacecraft. Geophys. Res. Lett. 35, L10110.

Rouillard, A.P., Savani, N., Davies, J.A., Lavraud, B., Forsyth, R.J., Morley, S.K., Opitz, A., Sheeley, N.R., Sauvaud, J.-A., Simunac, K.D.C., Luhmann, J.G., Galvin, A.G., Crothers, S.R., Davis, S.J., Harrison, R.A., Lockwood, M., Eyles, C.J., Bewsher, D., Brown, D.S.: 2009, A multispacecraft analysis of small scale transient entrained by solar wind streams. Solar Phys. this issue. doi:10.1007/s11207-009-9329-6.

Russell, C.T., Shinde, A.A.: 2003, ICME identification from solar wind ion measurements. Solar Phys. 216, $285-294$.

Sauvaud, J.-A., Larson, D., Aoustin, C., Curtis, D., Medale, J.-L., Fedorov, A., Rouzaud, J., Luhmann, J., Moreau, T., Schröder, P., Louarn, P., Dandouras, I., Penou, E.: 2008, The IMPACT Solar Wind Electron Analyzer (SWEA). Space Rev. Sci. 136, $227-239$.

Sheeley, N.R. Jr., Wang, Y.-M., Hawley, S.H., Brueckner, G.E., Dere, K.P., Howard, R.A., Koomen, M.J., Korendyke, C.M., Michels, D.J., Paswaters, S.E., Socker, D.G., St. Cyr, O.C., Wang, D., Lamy, P.L., Llebaria, A., Schwenn, R., Simnett, G.M., Plunkett, S., Biesecker, D.A.: 1997, Measurements of flow speeds in the corona between 2 and $30 R_{\odot}$. Astrophys. J. 484, 472-478.

Sheeley, N.R. Jr., Herbst, A.D., Palatchi, C.A., Wang, Y.-M., Howard, R.A., Moses, J.D., Vourlidas, A., Newmark, J.S., Socker, D.G., Plunkett, S.P., Korendyke, C.M., Burlaga, L.F., Davila, J.M., Thompson, W.T., St. Cyr, O.C., Harrison, R.A., Davis, C.J., Eyles, C.J., Halain, J.P., Wang, D., Rich, N.B., Battams, K., Esfandiari, E., Stenborg, G.: 2008a, Heliospheric images of the solar wind at Earth. Astrophys. J. 675, $853-862$.

Sheeley, N.R. Jr., Herbst, A.D., Palatchi, C.A., Wang, Y.-M., Howard, R.A., Moses, J.D., Vourlidas, A., Newmark, J.S., Socker, D.G., Plunkett, S.P., Korendyke, C.M., Burlaga, L.F., Davila, J.M., Thompson, W.T., St. Cyr, O.C., Harrison, R.A., Davis, C.J., Eyles, C.J., Halain, J.P., Wang, D., Rich, N.B., Battams, K., Esfandiari, E., Stenborg, G.: 2008b, SECCHI observations of the Sun's Garden-Hose density spiral. Astrophys. J. 674, L109-L112.

Wang, Y.-M., Sheeley, N.R. Jr.: 1990, Solar wind speed and coronal flux-tube expansion. Astrophys. J. 355, $726-732$.

Wang, Y.-M., Sheeley, N.R. Jr., Walters, J.H., Brueckner, G.E., Howard, R.A., Michels, D.J., Lamy, P.L., Schwenn, R., Simnett, G.M.: 1998, Origin of streamer material in the outer corona. Astrophys. J. 498, L165 - L168.

Wang, Y.-M., Sheeley, N.R. Jr., Socker, D.G., Howard, R.A., Rich, N.B.: 2000, The dynamical nature of coronal streamers. J. Geophys. Res. 105, $25133-25142$.

Yashiro, S., Gopalswamy, N., Michalek, G., St. Cyr, O.C., Plunkett, S.P., Rich, N.B., Howard, R.A.: 2004, A catalog of white light coronal mass ejections observed by the SOHO spacecraft. J. Geophys. Res. 109, A07105. doi:10.1029/2003JA010282.

Zhao, X.-P., Hundhausen, A.J.: 1983, Spatial structure of solar wind in 1976. J. Geophys. Res. 88, $451-454$.

Zurbuchen, T.H., Richardson, I.G.: 2006, In situ solar wind and magnetic field signatures of interplanetary coronal mass ejections. Space Sci. Rev. 123, $31-43$. 